\title{
Modified Quadratic Congruence Codes for Fiber Bragg-Grating-Based Spectral-Amplitude-Coding Optical CDMA Systems
}

\author{
Zou Wei, Student Member, IEEE, H. M. H. Shalaby, Senior Member, IEEE, and \\ H. Ghafouri-Shiraz, Senior Member, IEEE
}

\begin{abstract}
We have constructed a series of new code families for the spectral-amplitude-coding optical code-division multiple-access (CDMA) system, and proposed new transmitter and receiver structures based on tunable chirped fiber Bragg gratings (FBGs). The proposed system has been analyzed by taking into account the effects of phase-induced intensity noise, shot noise, and thermal noise. We have also compared the performance of this system with that of a former system where a Hadamard code is used. It has been shown that the new code families can suppress the intensity noise effectively and improve the system performance significantly. When the effective power is large (i.e., $>-10 \mathrm{dBm}$ ), the intensity noise is the main factor that limits the system performance. When the effective power is not sufficiently large, thermal and shot noise sources become the main limiting factors and the effect of thermal noise is much larger than that of shot noise.
\end{abstract}

Index Terms-Fiber Bragg gratings (FBGs), modified quadratic congruence (MQC) code, multiuser interference (MUI), optical code division multiple access (CDMA), spectral amplitude coding.

\section{INTRODUCTION}

$\mathbf{O}$ PTICAL code-division multiple-access (CDMA) systems are getting more and more attractive in the field of all-optical communications as multiple users can access the network asynchronously and simultaneously with high level of transmission security [1], [2]. Spectral-amplitude-coding (SAC) optical CDMA systems are now receiving more attention because the multiuser interference (MUI) can be canceled when code sequences with fixed in-phase cross correlation (such as $m$-sequence or Hadamard codes) are used [3]. Let $\lambda=\sum_{i=1}^{N} x_{i} y_{i}$ be defined as the in-phase cross correlation of two different sequences $X=\left(x_{1}, x_{2}, \ldots x_{N}\right)$ and $Y=$ $\left(y_{1}, y_{2}, \ldots y_{N}\right)$. We can denote a code by $(N, w, \lambda)$ where $N$ is the length, $w$ is the weight, and $\lambda$ is its in-phase cross correlation.

However, as broad-band thermal sources (BTS) are used in such systems, the phase-induced intensity noise (PIIN) that is due to the intensity fluctuation of BTS severely affects the

Manuscript received January 12, 2001; revised May 15, 2001.

Z. Wei and H. Ghafouri-Shiraz are with the School of Electrical and Electronic Engineering, Nanyang Technological University, Singapore 639798, Singapore.

H. M. H. Shalaby was with the School of Electrical and Electronic Engineering, Nanyang Technological University, Singapore 639798, Singapore. He is now with the Department of Electrical Engineering, University of Alexandria, Alexandria 21544, Egypt.

Publisher Item Identifier S 0733-8724(01)07750-7. system performance [4]. It has been shown that the effect of PIIN is proportional to the square of photocurrent and the system performance cannot be improved by simply increasing the received optical power [5]. To suppress PIIN's effect, a new code defined as $\left(\frac{q^{m+1}-1}{q-1}, \frac{q^{m}-1}{q-1}, \frac{q^{m-1}-1}{q-1}\right)$ has been introduced in [6] based on the theorem of block designs. It has been found that the intensity noise can be effectively suppressed by using this code and, hence, a higher signal-to-noise ratio (SNR) results. The higher SNR is, in fact, due to the higher ratio of the autocorrelation peak to the fixed in-phase cross correlation as compared with $m$-sequence or Hadamard code. However, no clear code construction approach was presented in [6]. In this paper, we have proposed a series of new $\left(p^{2}+p, p+1,1\right)$ codes for each prime number $p$ based on the quadratic congruence (QC) codes in [7]. It has been shown that these new codes have similar properties with the former code in [6].

In recent years, fiber Bragg gratings (FBGs) have been used to implement the encoder-decoder for optical CDMA. A novel coherent spectral phase-encoder composed of FBGs has been proposed in [8]. Similarly, we have designed both the transmitter and receiver structures for an SAC system based on our proposed new codes. We have also analyzed the performance by Gaussian approximation taking into account the effects of PIIN, shot noise, and thermal noise. The result has been compared with that of a former system where a Hadamard code is used [5].

It has been pointed out that when both the PIIN and shot noise are considered, the count of the arrived photons obeys negative binomial distribution [4]. If there is only one active user, the negative-binomial-distributed photoelectron counting will result in a lower bit error rate (BER) than Gaussian approximation because of its lower probability tails [9]. This result has also been verified experimentally by Dennis and Young [10]. However, in an optical CDMA system, the pulse hit (overlap) often occurs especially when the active user number is large. Therefore, it is reasonable to use Gaussian approximation in such a system.

The rest of this paper is organized as follows. Section II is devoted to the code construction and properties. Section III is devoted to the transmitter and receiver structures, as well as the discussions on the important aspects for the implementation of the designed system. In Section IV, we analyze the system performance taking account of intensity noise, shot noise, and thermal noise. Moreover, some numerical results are also presented. Finally, conclusions are drawn in Section V. 
TABLE I

SEQUENCES $y_{\alpha, \beta}(k)$ AND $s_{\alpha, \beta}(k)$ WHEN $p=5, d=1$, AND $b=2$

\begin{tabular}{c|c|c|c|cccccc}
\hline$\alpha$ & $\beta$ & $y_{\alpha, \beta}(k)$ & \multicolumn{7}{|c}{$s_{\alpha, \beta}(k)$} \\
\hline 0 & 0 & 014412 & 10000 & 01000 & 00001 & 00001 & 01000 & 00100 \\
\hline 1 & 0 & 144103 & 01000 & 00001 & 00001 & 01000 & 10000 & 00010 \\
\hline 4 & 0 & 101441 & 01000 & 100000 & 01000 & 00001 & 00001 & 01000 \\
\hline 1 & 3 & 422433 & 00001 & 00100 & 00100 & 00001 & 00010 & 00010 \\
\hline 3 & 4 & 304030 & 00010 & 100000 & 000001 & 100000 & 000110 & 100000 \\
\hline
\end{tabular}

\section{CODE CONSTRUCTION AND CODE PROPERTIES}

\section{A. Codes Construction}

The new proposed code families $\left(p^{2}+p, p+1,1\right)$, which are referred to as modified quadratic congruence (MQC) codes, can be constructed using the following steps.

- Step 1: We first construct a sequence of integer numbers as $y_{\alpha, \beta}(k)$ that are elements of a finite field $\mathrm{GF}(p)$ over an odd prime $p(p>2)$ by using the following expression:

$y_{\alpha, \beta}(k)=\left\{\begin{array}{l}{\left[d(k+\alpha)^{2}+\beta\right](\bmod p), \quad k=0,1, \ldots, p-1} \\ {[\alpha+b](\bmod p), \quad k=p}\end{array}\right.$

where $d \in\{1,2,3, \ldots, p-1\}$ and $b, \alpha, \beta \in$ $\{0,1,2, \ldots, p-1\}$. Each resulting sequence $y_{\alpha, \beta}(k)$ has $(p+1)$ elements, and we can generate $p^{2}$ different sequences for each pair of fixed parameters $d$ and $b$ by changing parameters $\alpha$ and $\beta$. These $p^{2}$ different sequences form a code family. Therefore, there are, in total, $p(p-1)$ code families when $d$ and $b$ change.

- Step 2: We next construct a sequence of binary numbers $(0,1)$ as $s_{\alpha, \beta}(k)$ based on the generated sequence $y_{\alpha, \beta}(k)$ by using the following mapping method:

$$
s_{\alpha, \beta}(i)= \begin{cases}1 & \text { if } i=k p+y_{\alpha, \beta}(k), \\ 0, & \text { otherwise }\end{cases}
$$

where $i=0,1,2, \ldots, p^{2}-1$ and $k=\left\lfloor\frac{i}{p}\right\rfloor$. Here, $\lfloor i / p\rfloor$ denotes the largest integer less than or equal to the value of $(i / p)$.

In Fig. 1, we have shown the procedure for generating $(0,1)$ sequence $s_{\alpha, \beta}(k)$ based on the number sequence $y_{\alpha, \beta}(k)$. We have also shown some code sequences in Table I for parameters $p=5, d=1$, and $b=2$.

\section{B. Properties of MQC Codes}

For a given fixed value of $b$, we can construct $(p-1)$ distinctive code families by using different values of parameter $d$. In each of these families, there are $p^{2}$ code sequences having following properties.

- Each code sequence has $p^{2}+p$ elements that can be divided into $(p+1)$ groups, and each group contains one " 1 " and $(p-1)$ " 0 "s.

- In-phase cross correlation $\lambda$ between any two sequences is always equal to 1 .

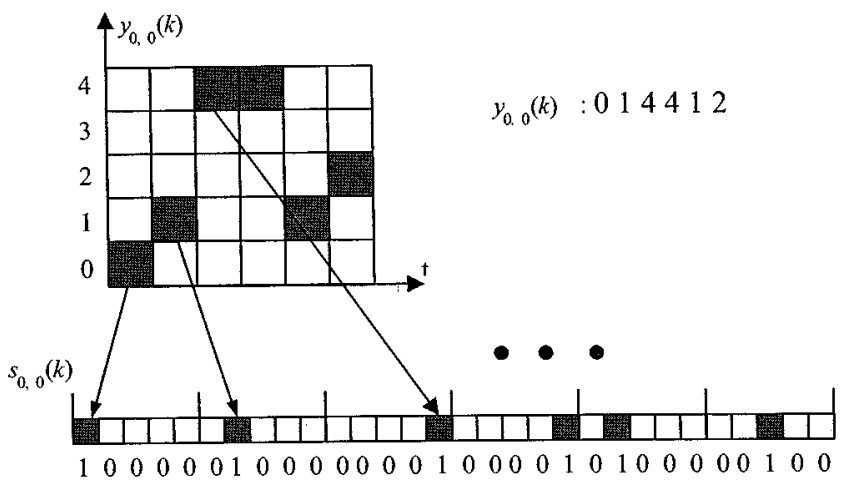

Fig. 1. Generation of $y_{0,0}(k)$ and $s_{0,0}(k)$ for $p=5, d=1, b=2$.

Because of these two properties, MQC codes are much better than Hadamard codes for the SAC optical CDMA systems. The first property can be easily obtained from the mapping method as explained in step 2, and the second one comes from the following definition and theorem.

Definition: Let $n$ be a fixed positive integer. Two integers $a$ and $b$ are said to be congruent modulo $n$, symbolized by $a \equiv$ $b(\bmod n)$, if $n$ divides the difference $a-b$; that is, provided that $a-b=k n$ for some integer $k$. For example, $3 \equiv 24(\bmod 7)$.

Theorem: Let $d$ and $b$ be constant numbers and $p$ be a prime number $(p>2)$. Then the following congruent equation has one incongruent solution except when $\alpha_{1}=\alpha_{2}$ and $\beta_{1}=\beta_{2}$ where there are $(p+1)$ incongruent solutions:

$$
y=y_{\alpha 1, \beta 1}(k)-y_{\alpha 2, \beta 2}(k)=0 .
$$

Proof: It should be noted that when $\left(\alpha_{1}=\alpha_{2}\right.$ and $\beta_{1}=$ $\left.\beta_{2}\right)$ the two sequences are the same. Therefore, $(p+1)$ incongruent solutions result in that case. To solve (3a) under other conditions, let us substitute (1) into it and consider the following two cases.

Case 1) When $k=0,1,2, \ldots, p-1$, then (3a) becomes

$$
2 d\left(\alpha_{1}-\alpha_{2}\right) k+d \alpha_{1}^{2}-d \alpha_{2}^{2}+\beta_{1}-\beta_{2}=0 \quad(\bmod p)
$$

where $d \in\{1,2,3, \ldots, p-1\}$ and $\alpha_{1}, \alpha_{2}, \beta_{1}$, $\beta_{2} \in\{0,1,2, \ldots, p-1\}$. Noting that $p>2$, this equation can be analyzed as follows. When $\alpha_{1} \neq$ $\alpha_{2}$, by Lagrange's theorem and its corollary, there exists only one solution given by

$$
k=\frac{d\left(\alpha_{2}^{2}-\alpha_{1}^{2}\right)+\beta_{2}-\beta_{1}}{2 d\left(\alpha_{1}-\alpha_{2}\right)} \quad(\bmod p) .
$$




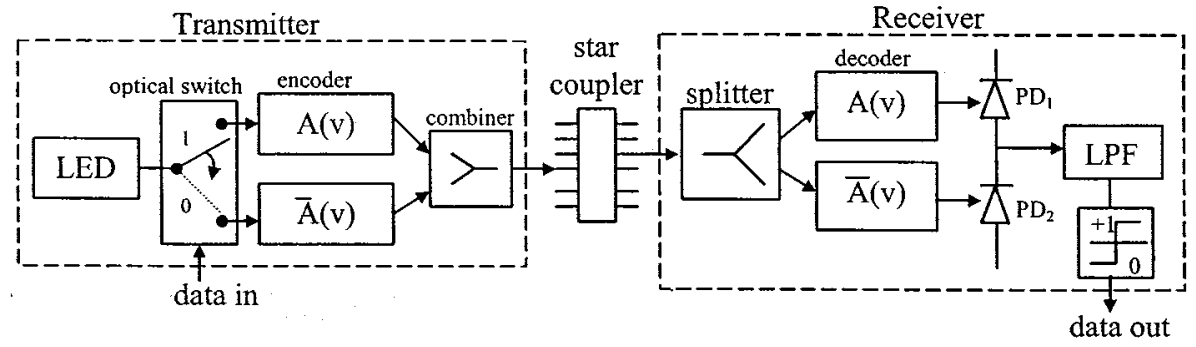

Fig. 2. Block diagram of the SAC optical CDMA system.

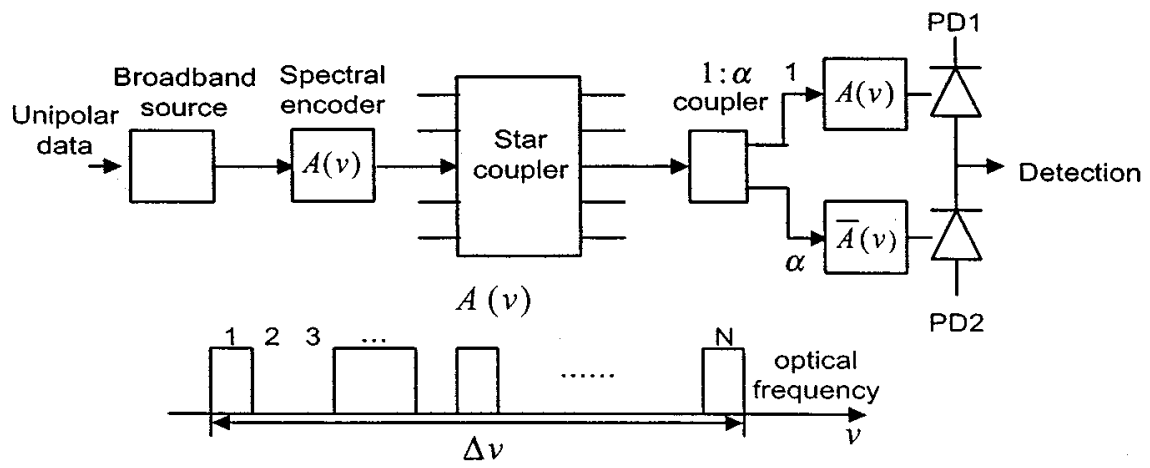

Fig. 3. Block diagram of SAC optical CDMA systems using MQC code.

When $\alpha_{1}=\alpha_{2}$ and $\beta_{1}=\beta_{2}$, there are $p$ solutions for $k$ in (3b) whereas when $\alpha_{1}=\alpha_{2}$ and $\beta_{1} \neq \beta_{2}$, no solution can be obtained.

Case 2) When $k=p$, (3a) becomes

$$
\alpha_{1}-\alpha_{2}=0 \quad(\bmod p) \text {. }
$$

This equation is valid only when $\alpha_{1}=\alpha_{2}$ and its solution is $k=p$.

Consequently, when $\alpha_{1}=\alpha_{2}$ and $\beta_{1}=\beta_{2}$, there are, in total, $(p+1)$ solutions of $k$ in (3a). Whereas when either $\left(\alpha_{1}=\alpha_{2}\right.$ and $\beta_{1} \neq \beta_{2}$ ) or $\alpha_{1} \neq \alpha_{2}$, (3a) has one solution of $k$. Therefore, there is exactly one solution for $k$ except if $\alpha_{1}=\alpha_{2}$ and $\beta_{1}=$ $\beta_{2}$ when there are $(p+1)$ incongruent solutions.

For a $(N, w, \lambda)$ code, the upper bound of the code size $A$ is given by [11]

$$
A(N, w, \lambda) \leq \frac{N(w-\lambda)}{w^{2}-N \lambda}, \quad w^{2}>N \lambda
$$

For MQC code, $N=p^{2}+p, w=p+1$, and $\lambda=1$, the upper bound of code size is $p^{2}$. Therefore, an MQC code that has $p^{2}$ code sequences in each family is an optimal code that we can obtain.

\section{System DESIGN AND Discussion}

\section{A. Former SAC System Using a Hadamard Code}

The block function of an SAC system using a Hadamard code is shown in Fig. 2 [5]. It should be noted that the used Hadamard code is unipolar because we can only detect the energy of an optical pulse. The detail procedures to generate a unipolar Hadamard code refer to [5]. For each bit of data, one broad-band optical pulse from the LED is encoded. The transmitter sends a pulse with spectral distribution $A(v)$ when the data bit is "1" and a pulse with complementary spectral distribution $\bar{A}(v)$ when the data bit is " 0 ." The shape of $A(v)$ is a sequence of spectral pulses arranged according to Hadamard code. All pulses from all users are mixed up at the star coupler, and then this superimposed signal is sent back to each user. At the receiver, the received optical signal is split and decoded by two complimentary decoders at first. Then, after balanced detection, low-pass filter (LPF), and threshold decision, the original data can be recovered. In [5], it has been shown that the MUI can be eliminated in such an optical CDMA system.

\section{B. An SAC System Using $\left(\frac{q^{m+1}-1}{q-1}, \frac{q^{m}-1}{q-1}, \frac{q^{m-1}-1}{q-1}\right)$ Code}

A detailed functional block diagram (see Fig. 3) has been proposed in [6] for the use of the above code in an SAC optical CDMA system. In this system, a pulse with specified spectral distribution is sent when the data bit is " 1 " and nothing is sent when the data bit is "0." At the receiver side, a 1: $\alpha$ splitter is used, at first, to divide the received signal into two parts. Then, they are input, respectively, into two decoders with complementary decoding functions. If $(N, w, \lambda)$ code is used, the MUI coming from $(k-1)$ undesired users at the first photodetector (PD1) is equal to $(k-1) \lambda$, and that at PD2 is equal to $\alpha(w-\lambda)(k-1)$. When $\alpha=\lambda /(w-\lambda)$, these two MUI components are equal. Therefore, after balanced photodetection, the effects of MUI can be canceled [6]. This functional block diagram can also be used for MQC codes because they have fixed in-phase cross correlation, just like the code in [6].

\section{Structure of Transmitter and Receiver Based on FBG}

Figs. 4 and 5 show our proposed transmitter and receiver structures (based on FBGs and MQC codes), respectively, for SAC optical CDMA. When a broad-band pulse is input into a group of FBGs, the spectral components corresponding to $A(v)$ 


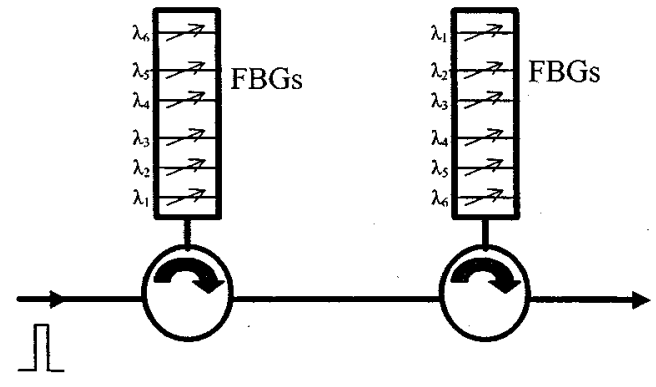

Fig. 4. Encoder $(p=5)$.

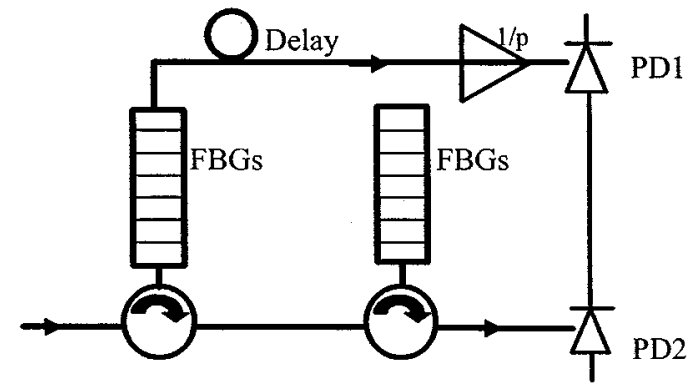

Fig. 5. Decoder $(p=5)$.

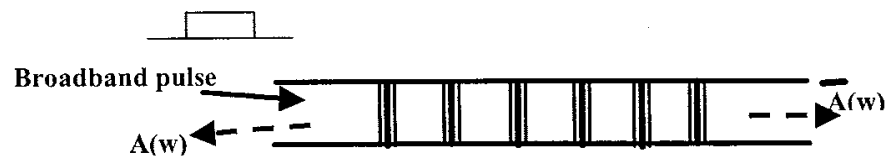

Fig. 6. Graphic representation of the FBG's function.

will be reflected back, and the output at the other end of the grating group will contain all the complementary components corresponding to $\bar{A}(v)$ (see Fig. 6). In the receiver shown in Fig. 5, the output from the top of the first FBG group is used directly as the decoded output $R(v) \bar{A}_{k}(v)$, where $R(v)$ is the received signal and $\bar{A}_{k}(v)$ is the complement of the receiver address sequence. In this way, we can utilize all the received optical power efficiently without any need to consider the loss incurred by the $1: \alpha$ coupler shown in Fig. 3 .

When bit "1" is sent, an optical pulse from a broad-band thermal source (BTS) launches into the encoder, whereas no optical pulse is sent if the data bit is " 0. ." The optical pulse passes through the first fiber-grating groups and correspondent spectral components are reflected. For the reconfiguration of the destined address code, the gratings in the encoder are all tunable, which means the central wavelength of the reflected spectral component of each grating can be changed. The second group of FBGs in the transmitter is used to compensate the round-trip delay of different spectral components so that all the reflected components have the same time delay and can be incorporated into a pulse again. At the receiver, each grating is fixed according to the receiver's address. MUI can be canceled by balanced detection because the in-phase cross correlation between any two code-sequences is always equal to 1 .

It should be noted that it is unnecessary to divide the source spectrum precisely because, in such a system, each transmitted pulse is generated by the reflection of a fiber grating and, hence, at the receiver, the power of original data pulse will be completely reflected when the grating is sufficiently long.

Due to the lower weight of MQC codes, we can construct a transmitter-receiver with only a few FBGs. Therefore, it is very easy to implement. However, if Hadamard codes are used for the same purpose, we may need too many FBGs.

\section{Consideration of Tunable Range and Bit-Rate Limitation}

Grating tuning can be implemented by fiber stretching using piezoelectric devices or by temperature adjustment. When MQC codes are used, due to its first property, the required spectral tunable range (changing range by tuning) for each grating is equal to $1 /(p+1)$ of the total utilized bandwidth. Therefore, we can greatly reduce the required tunable range for each grating by using MQC codes. A grating's tunable linewidth range $\Delta \lambda_{s}$ can be expressed as $\Delta \lambda_{s}=0.8 \lambda \Delta L / L$, where $L$ is the grating length and $\Delta L$ is its change by stretching [12]. Let $\Delta L / L=$ 0.005 and operating wavelength $\lambda=1550 \mathrm{~nm}$, the tunable linewidth range is given by $\Delta \lambda_{s}=6.2 \mathrm{~nm}$. Because there are $(p+1)$ gratings in each encoder, we can utilize 37-nm linewidth if $p=5$. On the other hand, when the total linewidth is $30 \mathrm{~nm}$, the required value of $\Delta L / L$ can be reduced to 0.004 . Therefore, the use of MQC codes makes it more convenient to realize the encoder reconfiguration.

In the FBG-based encoding-decoding structure, the length of the FBG group no longer limits the bit rate, as was the case in [12]. This is because the delay of each spectral component is the same after delay compensation. Therefore, the bit rate is mainly limited by two factors: i) the maximal modulation rate of the BTS and ii) the bandwidths of the photodetectors (PDs) used in the balanced detection.

\section{System Performance ANALysis}

In the analysis of the proposed system (see Figs. 4 and 5), we have considered incoherent intensity noise, as well as shot and thermal noises in both PDs. The effect of the receiver's dark current is neglected. Gaussian approximation is used for the calculation of BER.

The variance of photocurrent due to the detection of an ideally unpolarized thermal light, which is generated by spontaneous emission, can be expressed as

$$
\left\langle i^{2}\right\rangle=2 e I B+I^{2} B \tau_{c}+4 K_{b} T_{n} B / R_{L},
$$

where

$e \quad$ electron's charge;

$I$ average photocurrent;

$B$ noise-equivalent electrical bandwidth of the receiver;

$\tau_{c} \quad$ coherence time of source;

$K_{b} \quad$ Boltzmann's constant;

$T_{n} \quad$ absolute receiver noise temperature;

$R_{L}$ receiver load resistor.

In this equation, the first item results from the shot noise, the second item denotes the effect of PIIN [5], and the third item represents the effect of thermal noise. Let $G(v)$ be the single 
sideband power spectral density (PSD) of the source. Its coherence time can be expressed as [5]

$$
\tau_{c}=\frac{\int_{0}^{\infty} G^{2}(v) d v}{\left[\int_{0}^{\infty} G(v) d v\right]^{2}}
$$

In (5), the total effect of PIIN and shot noise obeys negative binomial distribution [4], and thermal noise has a Gaussian distribution. To simplify our analysis, Gaussian approximation is used for all of them. In the following analysis, we have considered the effects of both shot and thermal noises, as well as the effect of PIIN. Let $c_{k}(i)$ denote the $i$ th element of the $k$ th MQC code sequence. The code properties can be written as

$$
\begin{aligned}
& \sum_{i=1}^{N} c_{k}(i) c_{l}(i)= \begin{cases}p+1, & k=l \\
1, & k \neq l\end{cases} \\
& \text { and } \\
& \sum_{i=1}^{N} c_{k}(i) \bar{c}_{l}(i)= \begin{cases}0, & k=l \\
p, & k \neq l\end{cases}
\end{aligned}
$$

To analyze the system with transmitter and receiver shown in Figs. 4 and 5, we assume the following.

i) Each light source is ideally unpolarized and its spectrum is flat over the bandwidth $\left[v_{0}-\Delta v / 2, v_{0}+\Delta v / 2\right]$, where $v_{0}$ is the central optical frequency and $\Delta v$ is the optical source bandwidth in hertz.

ii) Each power spectral component has identical spectral width.

iii) Each user has equal power at the receiver.

iv) Each bit stream from each user is synchronized.

Based on the above assumptions, we can easily analyze the system performance using Gaussian approximation. The PSD of the received optical signals can be written as

$$
\begin{aligned}
r(v)=\frac{P_{s r}}{\Delta v} \sum_{k=1}^{K} d_{k} \sum_{i=1}^{N} c_{k}(i)\{ & u\left[v-v_{0}-\frac{\Delta v}{2 N}(-N+2 i-2)\right] \\
& \left.-u\left[v-v_{0}-\frac{\Delta v}{2 N}(-N+2 i)\right]\right\}
\end{aligned}
$$

where $P_{s r}$ is the effective power of a broad-band source at the receiver (if the loss due to transmission and star coupler is $\xi$ and power of source pulse is $P_{o}$, then the received effective power is $\left.\xi P_{o}\right), K$ is the number of active users less than or equal to $p^{2}$, $N$ is the MQC code length given by $N=p^{2}+p, d_{k}$ is the data bit of the $k$ th user that is " 1 " or " 0 ," and $u(v)$ is the unit step function expressed as

$$
u(v)= \begin{cases}1, & v \geq 0 \\ 0, & v<0 .\end{cases}
$$

Therefore, the PSD at PD1 and PD2 (see Fig. 5) of the $l$ th receiver during one bit period can be written as

$$
\begin{aligned}
G_{1}(v)= & \frac{1}{p} \frac{P_{s r}}{\Delta v} \sum_{k=1}^{K} d_{k} \sum_{i=1}^{N} c_{k}(i) \bar{c}_{l}(i) \\
& \cdot\left\{u\left[v-v_{0}-\frac{\Delta v}{2 N}(-N+2 i-2)\right]\right. \\
& \left.\quad-u\left[v-v_{0}-\frac{\Delta v}{2 N}(-N+2 i)\right]\right\}
\end{aligned}
$$

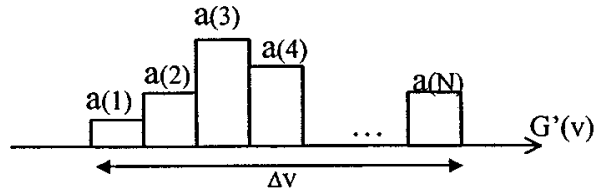

Fig. 7. The PSD of the received signal $r(v)$.

$$
\begin{aligned}
G_{2}(v)= & \frac{P_{s r}}{\Delta v} \sum_{k=1}^{K} d_{k} \sum_{i=1}^{N} c_{k}(i) c_{l}(i) \\
\cdot & \left\{u\left[v-v_{0}-\frac{\Delta v}{2 N}(-N+2 i-2)\right]\right. \\
& \left.-u\left[v-v_{0}-\frac{\Delta v}{2 N}(-N+2 i)\right]\right\}
\end{aligned}
$$

and then

$$
\begin{aligned}
& \int_{0}^{\infty} G_{1}(v) d v=\frac{P_{s r}}{N} \sum_{k=1, k \neq l}^{K} d_{k} \\
& \int_{0}^{\infty} G_{2}(v) d v=\frac{P_{s r}}{N}(p+1) d_{l}+\frac{P_{s r}}{N} \sum_{k=1, k \neq l}^{K} d_{k} .
\end{aligned}
$$

To calculate the integral of $G_{1}^{2}(v)$ and $G_{2}^{2}(v)$, let us first consider an example of the PSD [denoted by $G^{\prime}(v)$ ] of the received superimposed signal, which is shown in Fig. 7, where $a(i)$ is the amplitude of the $i$ th spectral slot with width $(\Delta v / N)$. The integral of $G^{\prime 2}(v)$ can be expressed as

$$
\int_{0}^{\infty} G^{2}(v) d v=\frac{\Delta v}{N} \sum_{i=1}^{N} a^{2}(i) .
$$

Therefore, using (10) and (11), we have

$$
\begin{aligned}
\int_{0}^{\infty} G_{1}^{2}(v) d v= & \frac{1}{p^{2}} \frac{P_{s r}^{2}}{N \Delta v} \\
& \cdot \sum_{i=1}^{N}\left\{\bar{c}_{l}(i) \cdot\left[\sum_{k=1}^{K} d_{k} c_{k}(i)\right] \cdot\left[\sum_{m=1}^{K} d_{m} c_{m}(i)\right]\right\}
\end{aligned}
$$

$$
\begin{aligned}
\int_{0}^{\infty} G_{2}^{2}(v) d v= & \frac{P_{s r}^{2}}{N \Delta v} \\
& \cdot \sum_{i=1}^{N}\left\{c_{l}(i) \cdot\left[\sum_{k=1}^{K} d_{k} c_{k}(i)\right] \cdot\left[\sum_{m=1}^{K} d_{m} c_{m}(i)\right]\right\} .
\end{aligned}
$$

In the above equations, $d_{k}$ is the data bit of the $k$ th user that is either " 1 " or " 0. ." Consequently, the photocurrent $I$ can be expressed as

$$
\begin{aligned}
I=I_{2}-I_{1} & =\Re \int_{0}^{\infty} G_{2}(v) d v-\Re \int_{0}^{\infty} G_{1}(v) d v \\
& =\Re \frac{P_{s r}}{p} d_{l}
\end{aligned}
$$

where $\Re$ is the responsivity of the PDs given by $\Re=(\eta e) /\left(h v_{c}\right)$. Here, $\eta$ is the quantum efficiency, $e$ is the electron's charge, $\mathrm{h}$ is the Plank's constant, and $v_{c}$ is the central frequency of the original broad-band optical pulse. Because the noises in PD1 and PD2 are independent, the power 
of noise sources that exist in the photocurrent can be written as

$$
\begin{aligned}
\left\langle I^{2}\right\rangle & =\left\langle I_{1}^{2}\right\rangle+\left\langle I_{2}^{2}\right\rangle+\left\langle I_{t h}^{2}\right\rangle \\
& =2 e B\left(I_{1}+I_{2}\right)+B I_{1}^{2} \tau_{c 1}+B I_{2}^{2} \tau_{c 2}+4 K_{b} T_{n} B / R_{L} .
\end{aligned}
$$

Therefore

$$
\begin{aligned}
\left\langle I^{2}\right\rangle= & 2 e B \Re\left[\int_{0}^{\infty} G_{1}(v) d v+\int_{0}^{\infty} G_{2}(v) d v\right] \\
& +B \Re^{2} \int_{0}^{\infty} G_{1}^{2}(v) d v+B \Re^{2} \int_{0}^{\infty} G_{2}^{2}(v) d v \\
& +4 K_{b} T_{n} B / R_{L} .
\end{aligned}
$$

When all the users are transmitting bit "1," using the average value as $\sum_{k=1}^{K} c_{k}(i) \approx K / p$ and the correlation properties, the noise power can be written as

$$
\begin{array}{r}
\left\langle I^{2}\right\rangle=\frac{B \Re^{2} P_{s r}^{2} K}{\Delta v(p+1) p^{2}}\left(\frac{K-1}{p}+p+K\right) \\
+2 e B \Re P_{s r}\left[\frac{p-1+2 K}{p^{2}+p}\right]+4 K_{b} T_{n} B / R_{L} .
\end{array}
$$

Noting that the probability of sending bit " 1 " at any time for each user is $1 / 2$, the above equation becomes

$$
\begin{aligned}
& \left\langle I^{2}\right\rangle \cong \frac{B \Re^{2} P_{s r}^{2} K}{2 \Delta v(p+1) p^{2}}\left(\frac{K-1}{p}+p+K\right) \\
& \quad+e B \Re P_{s r}\left[\frac{p-1+2 K}{p^{2}+p}\right]+4 K_{b} T_{n} B / R_{L} .
\end{aligned}
$$

From (16) and (18), we can get the average SNR, as in (19) at the bottom of the page. Using Gaussian approximation, the BER can be expressed as

$$
P_{e}=\frac{1}{2} \operatorname{erfc}(\sqrt{\mathrm{SNR} / 8})
$$

Fig. 8 shows the relation between the number of simultaneous users and the SNR when the prime number used for MQC code construction is $p=7, p=11$, and $p=13$, respectively. The parameters used in our analysis are listed in Table II. The SNR curve of the former system using Hadamard codes (with the same effective pulse power and optical bandwidth) is also shown as a reference. In this figure, the effective power from each user is $-10 \mathrm{dBm}$ and the intensity noise is the main noise. It should be noted that the SNR values plotted in this figure are all average values, and each curve ends at the point where the number of simultaneous users is equal to the code size. It has been shown that MQC codes give a much higher SNR when the effective power is large. With a bigger prime number $p$, not only the number of accommodatable simultaneous users is significantly increased, but also a higher SNR results. Therefore, MQC codes can effectively suppress the effect of intensity noise and, hence, result in a much better performance. This suppression, in fact, comes from the lower in-phase cross correlation of MQC codes than that of Hadamard codes. Although MQC codes have a lower autocorrelation due to its lower weight, its

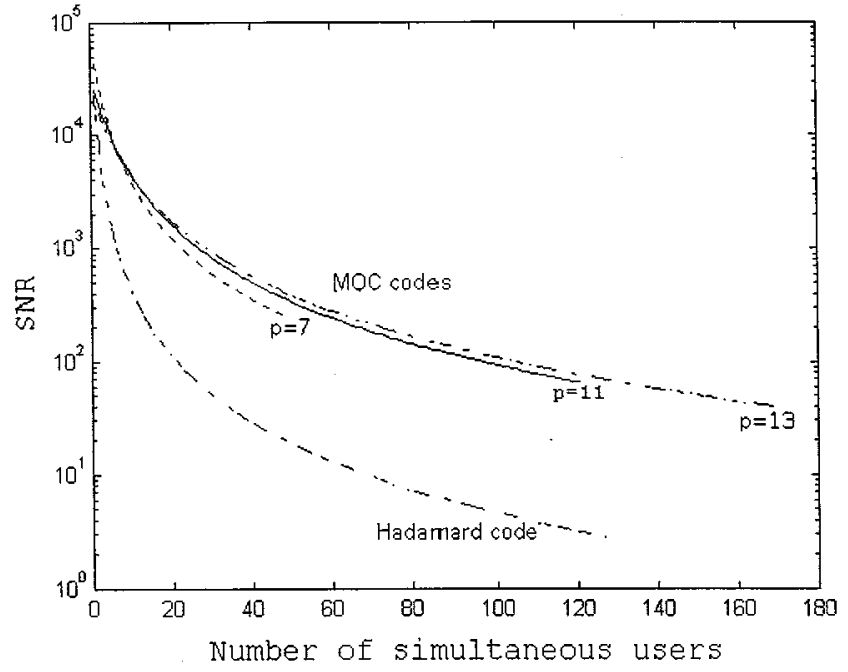

Fig. 8. SNRs versus number of simultaneous users when $P_{s r}=-10 \mathrm{dBm}$.

TABLE II

TYPICAL PARAMETERS USED IN THE CALCULATION

\begin{tabular}{l|l}
\hline PD quantum efficiency & $\eta=0.6$ \\
\hline Line-width of the thermal source & $\Delta v=3.75 \mathrm{THz}$ \\
\hline Operating wavelength & $\lambda_{0}=1.55 \mu \mathrm{m}$ \\
\hline Electrical bandwidth & $\mathrm{B}=80 \mathrm{MHz}$ \\
\hline Data bit rate & $R_{b}=155 \mathrm{Mbps}$ \\
\hline Receiver noise temperature & $T_{r}=300 \mathrm{~K}$ \\
\hline Receiver load resistor & $R_{L}=1030 \Omega$ \\
\hline
\end{tabular}

autocorrelation-to-inphase-cross-correlation ratio $(w / ?)$ is still larger. However, this lower autocorrelation will cause the performance degradation when other noises sources are considered, especially at low signal power, as we will show in Fig. 11.

Fig. 9 shows variations of the BER with the number of simultaneous users when $P_{s r}=-10 \mathrm{dBm}$. For comparison, we have also included the BER variations of the former system that uses Hadamard codes. In the former system, because we also send a pulse when the data bit is " 0 ," the formula used to calculate the BER becomes $P_{e}=(1 / 2) \operatorname{erfc}(\sqrt{\mathrm{SNR} / 2})$. It has been clearly shown that the system using MQC codes has a much lower BER than the one that uses Hadamard codes.

Fig. 10 shows the BER variations with the effective power $P_{s r}$ when $p=7$ and the number of simultaneous users is 49 . The solid lines represent the BERs, taking into account effects of intensity, shot, and thermal noises. The dashed lines indicate the BER performances when effects of only intensity and shot noises are considered. The dotted lines indicate the system BERs when only intensity and thermal noise sources are considered. It is shown that, when $P_{s r}$ is large, both the shot and

$$
\begin{aligned}
\mathrm{SNR} & =\frac{\left(I_{2}-I_{1}\right)^{2}}{\left\langle I^{2}\right\rangle} \\
& =\frac{\Re^{2} P_{s r}^{2} / p^{2}}{B \Re^{2} P_{s r}^{2} K[(K-1) / p+p+K] /\left[2 \Delta v(p+1) p^{2}\right]+e B \Re P_{s r}(p-1+2 K) /\left(p^{2}+p\right)+4 K_{b} T_{n} B / R_{L}} .
\end{aligned}
$$




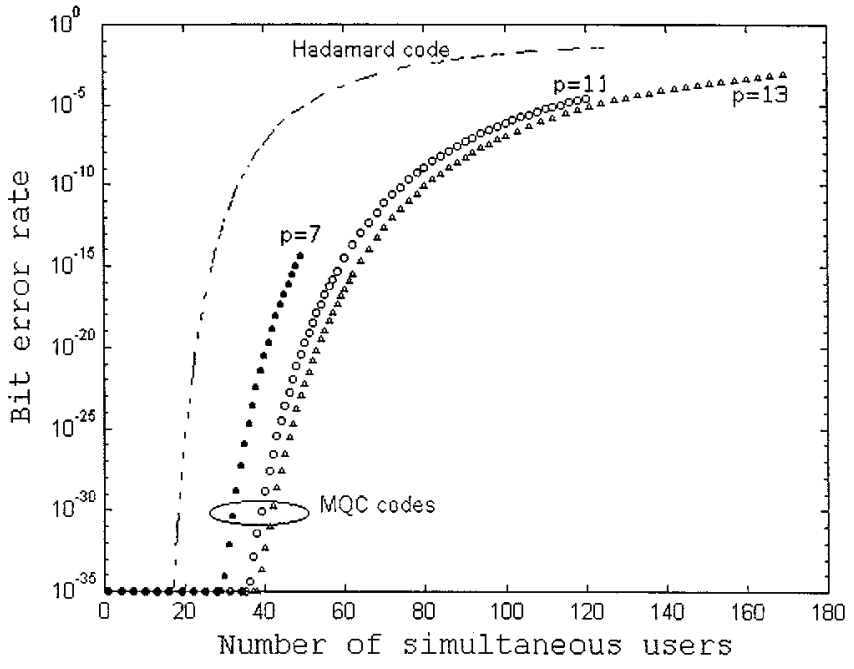

Fig. 9. BER versus number of simultaneous users when $P_{s r}=-10 \mathrm{dBm}$.

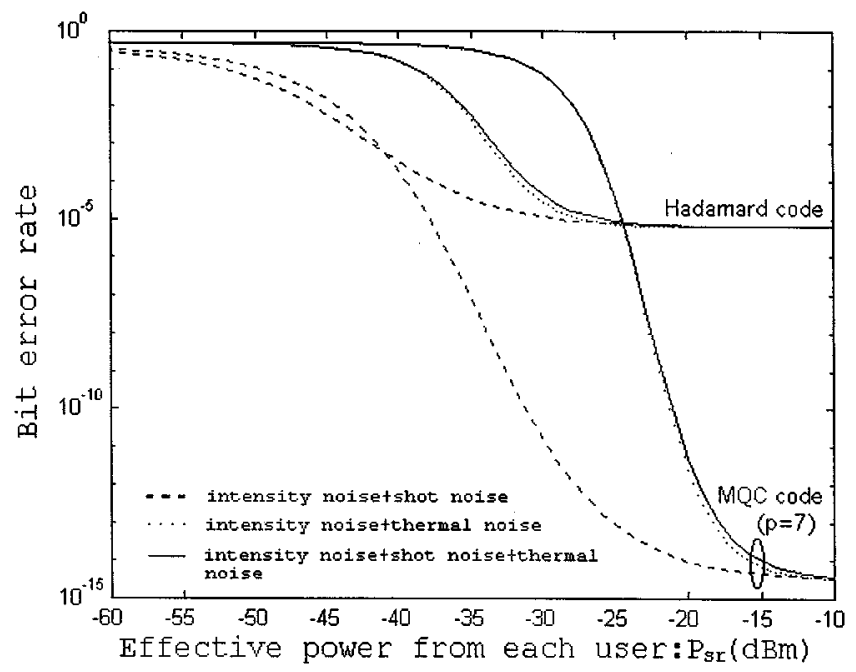

Fig. 10. BER versus effective power $P_{s r}$ when number of simultaneous users is 49 .

thermal noises are negligibly small compared with the intensity noise, which becomes the main limitation factor of the system performance. However, when $P_{s r}$ is low, the effect of intensity noise becomes minimal, and, hence, the thermal noise source becomes the main factor that limits the system performance. It is also shown that thermal noise is much more influential than shot noise on system performance.

Fig. 11 shows the BER variations with $P_{s r}$ when the number of simultaneous users is 49 and $p=7, p=11$, and $p=13$. The performance of the former system using Hadamard code is also shown for comparison. Fig. 12 shows variations of the BER versus number of simultaneous users for different values of $P_{s r}$. In these two figures, we have considered effects of the intensity, shot, and thermal noise sources. It is shown that when $P_{s r}$ is less than $-25 \mathrm{dBm}$, the former system using Hadamard codes will have better performance than the system using MQC codes. This is because the large value of prime number $p$ causes a large power loss $(=1 / p)$ in the transmitter, and, hence, the shot and thermal noise sources will severely affect the system performance, especially when $P_{s r}$ is not sufficiently large. However,

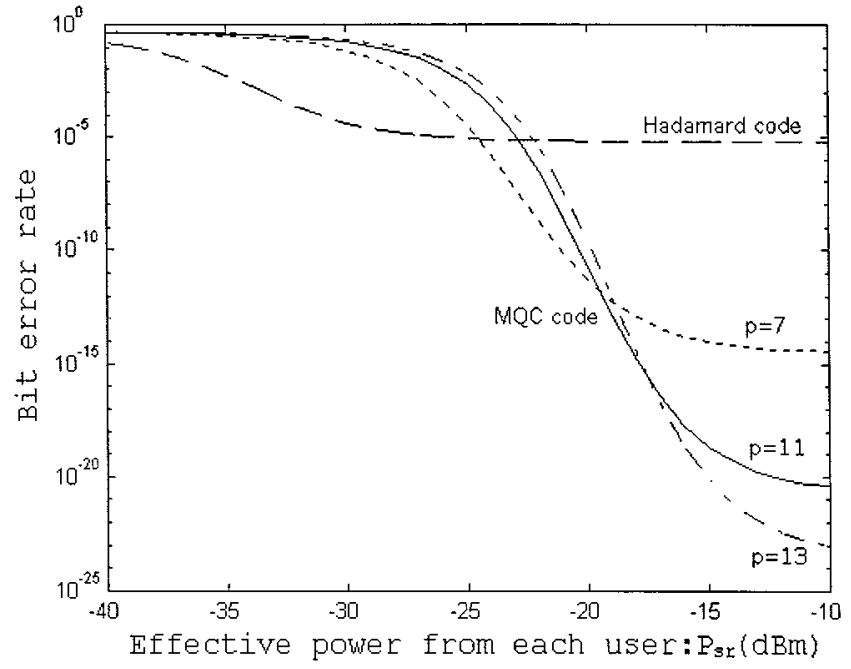

Fig. 11. BER versus effective power $P_{s r}$ when number of simultaneous users is 49 , taking into account the intensity noise, shot noise, and thermal noise.

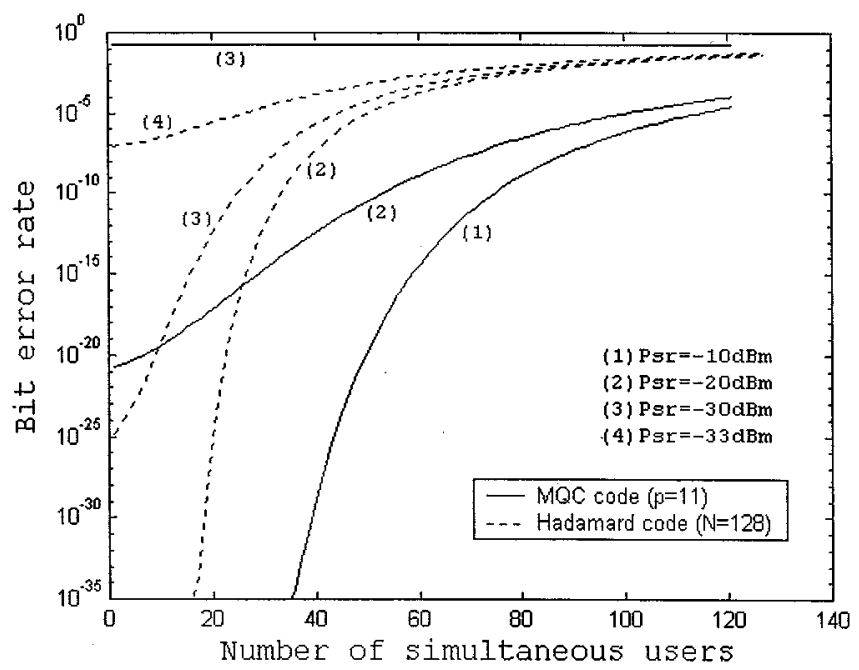

Fig. 12. BER versus number of simultaneous users when $P_{s r}$ is different.

the power loss incurred by Hadamard-code encoding is always $1 / 2$, regardless of the code length.

If we use an array of $(p+1)$ tunable lasers, we can provide large optical power even after the MQC encoder. In this case, the required tunable range of each laser is only $1 /(p+1)$ of the total optical bandwidth. This method does not have any limitation on the shape of PSD, as in the case of thermal source, and we can also enlarge the optical encoded bandwidth freely to get a higher SNR. Therefore, although the coherent interference between the overlapped optical pulses with the same wavelength may cause degradation, a better performance is promising in such a laser-array system.

\section{CONCLUSION}

In this paper, we have proposed a construction method for a series of new code families with a fixed in-phase cross correlation value of 1 . The properties of these codes have been proved and discussed. New structures of both the transmitter and receiver for the SAC optical CDMA system have been designed using FBG groups. The performance of the system with 
the new transmitter and receiver structures has been analyzed using MQC codes and taking into account effects of the intensity, shot, and thermal noise sources. The BER performances of both this system and a former one using Hadamard code have been compared. It has been shown that the new code families can suppress intensity noise effectively and improve the system performance significantly. When $P_{s r}$ is large, the intensity noise is the main factor that limits the system performance. However, when $P_{s r}$ is not sufficiently large, thermal and shot noise sources become the main limiting factors, and the effect of thermal noise is much lager than that of shot noise.

\section{REFERENCES}

[1] J. A. Salehi, "Code division multiple access techniques in optical fiber network-Part I: Fundamental principles," IEEE Trans. Commun., vol. 37, pp. 824-833, Aug. 1989.

[2] J. A. Salehi and C. A. Brackett, "Code division multiple access techniques in optical fiber network-Part II: System performance analysis," IEEE Trans. Commun., vol. 37, pp. 834-842, Aug. 1989.

[3] M. Kavehrad and D. Zaccarina, "Optical code-division-multiplexed system based on spectral encoding of noncoherent sources," J. Lightwave Technol., vol. 13, pp. 534-545, Mar. 1995.

[4] J. W. Goodman, Statistical Optics. New York: Wiley, 1985.

[5] E. D. J. Smith, R. J. Blaikie, and D. P. Taylor, "Performance enhancement of spectral-amplitude-coding optical CDMA using pulse-position modulation," IEEE Trans. Commun., vol. 46, pp. 1176-1185, Sept. 1998.

[6] X. Zhou, H. M. H. Shalaby, C. Lu, and T. Cheng, "Code for spectral amplitude coding optical CDMA systems," Electron. Lett., vol. 36, pp. 728-729, Apr. 13, 2000.

[7] Z. Kostic and E. L. Titlebaum, "The design and performance analysis for several new classes of codes for optical synchronous CDMA and for arbitrary-medium time-hopping synchronous CDMA communication systems," IEEE Trans. Commun., vol. 42, pp. 2608-2617, Aug. 1994

[8] A. Grunnet-Jepsen, A. E. Johnson, E. S. Maniloff, T. W. Mossberg, M. J. Munroe, and J. N. Sweetser, "Fiber Bragg grating based spectral encoder/decoder for lightwave CDMA," Electron. Lett., vol. 35, pp. 1096-1097, June 24, 1999.

[9] L. Nguyen, J. F. Young, and B. Aazhang, "Photoelectric current distribution and bit error rate in optical communication systems using a superfluorescent fiber source," J. Lightwave Technol., vol. 14, pp. 1455-1466, June 1996.

[10] T. Dennis and J. F. Young, "Measurements of BER performance for bipolar encoding of an SFS," J. Lightwave Technol., vol. 17, pp. 1542-1546, Sept. 1999.

[11] X. Zhou, H. M. H. Shalaby, and C. Lu, "Design and performance analysis of a new code for spectral-amplitude-coding optical CDMA systems," in IEEE 6th Int. Symp. Spread Spectrum Techniques Applications, vol. 1, 2000, pp. 174-178.

[12] H. Fathallah, L. A. Rusch, and S. LaRochelle, "Passive optical fas frequency-hop CDMA communication system," J. Lightwave Technol., vol. 17, pp. 397-405, Mar. 1999.

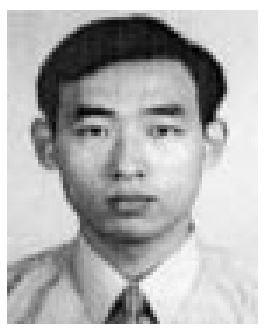

Zou Wei (S'01) was born in China in September 1973. He received the B.E. and M.E. degrees in telecommunication engineering from Beijing University of Posts and Telecommunications, Beijing, China, in 1995 and 1998, respectively. He is currently working toward the Ph.D. degree at the School of Electrical and Electronic Engineering at Nanyang Technological University, Singapore, Singapore.

In 1998, he joined Shanghai Telecommunications Research Center, Ministry of Posts and Telecommunications, Shanghai, China, where was engaged in research and development on common channel signaling and computer-telecommunication integration. His research interests include optical CDMA, digital signal processing, asynchronous transfer mode, and dense wavelength division multiplexing.

H. M. H. Shalaby (S'83-M'83-SM'99), photograph and biography not available at the time of publication.

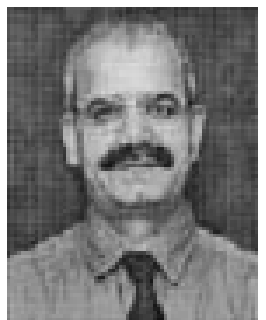

H. Ghafouri-Shiraz (S'85-M'86-SM'88) received the B.Sc. and M.Sc. degrees in electronic and electrical engineering form Shiraz University, Shiraz, Iran, in 1973 and 1978, respectively, and the D.Eng. degree in electronic engineering from the University of Tokyo, Tokyo, Japan, in 1985.

From 1973 to 1980, he was an Assistant Professor at the Technical School of Electronics at Shiraz University, and from 1976, he was the Head of the Communication Department. From 1980 to 1981, he was the Head of the Communication and Maintenance Department at Shiraz Univeristy. From 1981 to 1985, he received the Japanese government scholarship (Monbusho) and was with the Electronics Department of the University of Tokyo, where he was involved in researching optical frequency-domain reflectometry. From 1985 to 1987, he was a Senior Research Fellow in the second research laboratory, optoelectronic development division of Anritsu Corporation, Tokyo, Japan. From 1987 to 1999, he has been a member of the academic staff at the School of Electronic and Electrical Engineering, University of Birmingham, Birmingham, U.K. His administrative responsibilities at the University of Birmingham were as Director of service courses, Director of the M.Sc. program in communication engineering, and member of the management and steering committees and the Board of Faculty of Engineering. In March 1999, Napier University, Edinburgh, U.K., short-listed and subsequently offered him the position of readership. In October 1999, he was awarded three years of absence from the University of Birmingham and he joined the School of Electrical and Electronic Engineering at Nanyang Technological University, Singapore, Singapore, as an Associate Professor. His research interests include optical CDMA, optical networks, optical devices, optical fiber communications, nonlinear optics, and microwave. In 1994, he served as the Guest Editor for special issues in Fibre and Integrated Optics and Microwave and Optical Technology Letters. He chaired some sessions of the Fourth International Conference on Millimeter Wave and Far Infrared Science and Technology in Beijing, China, in 1996 and an IEE colloquium in 1999. He has supervised many Ph.D. students and research fellows. He has published more than 152 papers in refereed journals, as well as the books Fundamentals of Laser Diode Amplifiers and Distributed Feedback Laser Diodes: Principles and Physical Modelling. He is a chartered engineer and a reviewer for several journals, including IEEE TRANSACTIONS and IEE Proceedings.

Dr. Ghafouri-Shiraz is a member of the IEE Professional Group on Optoelectronics. 